\title{
Die Kunst des Arztes und die Philosophie: Zur Genese eines Grundverhältnisses des europäischen Denkens
}

\begin{abstract}
The Art of Medicine and Philosophy: On the Genesis of a Basic Relationship in European Thought. Referring to the examples of Hippocrates and Socrates, in this essay, we establish the thesis that philosophy and medicine in Greek philosophy are to be regarded as strongly interdependent. In their view, interpretations of health and disease are intertwined with various contexts or settings such as living conditions, environment and climate, which has implications for the therapy of patients as an art of healing. The relevance and philosophical perspectives of this epoch for modern medicine and public health on a globalized planet are highlighted.
\end{abstract}

Einer der eigentlichen Beweggründe des Denkens der Antike ist der Versuch, dem Wunsch zu entsprechen, sich um die Welt zu sorgen und sie zu heilen, indem die Menschen dazu gebracht werden, die Zusammenhänge dieser Welt besser zu verstehen, d.h. die Welt in ihrer Ordnung (kosmos) genauer zu kennen und dank dieses Wissens im Einklang (harmonia) mit ihr zu sein. Dies setzt freilich schon eine Differenzierung des antiken Menschen hinsichtlich der gewachsenen Welt (physis) voraus. Diese zeigt sich in der Sesshaftigkeit der Gemeinschaft (polis) und den damit einhergehenden Zivilisationsproblemen seit der neolithischen Kultur. Der bereits vollzogene Bruch mit der physis, bei einer gleichzeitig immer noch sehr starken geistigen, religiösen und materiellen Kontextualisierung mit ihr, warf implizit die Frage nach einer Heilung von polis und physis, auf. Die frühen griechischen Denker, angefangen bei den Orphikern, Homer und Hesiod, dachten und dichteten darüber. ${ }^{1}$ Vor diesem Kontext erscheint es wenig verwunderlich, dass

1 Dies ist besonders präsent in Hesiods Werke und Tage, wo er mit den Mythen vom Feuer des Prometheus (Iapetosson) und dem Fass (wörtliche Übersetzung, ansonsten „Büchse“) der Pandora, die bekanntlich Krankheit, Verderben und die Hoffnung enthält, diese Ambivalenz von Natur und Technik, vom ursprünglichem Leben in der Natur und Zivilisationsproblemen an seinem geschichtlichen Ort bereits zum Thema macht (Hesiod 1996, S. 7-11).

Michael Medzech, Philosophisch-Theologische Hochschule Vallendar (PTHV)

Alexander Krämer, Fakultät für Gesundheitswissenschaften, Universität Bielefeld

Ә OpenAccess. (c) 2021 Michael Medzech und Alexander Krämer, publiziert von De Gruyter.

(cc) BY-NC-SA Dieses Werk ist lizenziert unter einer Creative Commons Namensnennung - Nicht kommerziell - Weitergabe unter gleichen Bedingungen 4.0 International Lizenz.

https://doi.org/10.1515/9783110713336-007 
für einige Philosophen der Antike die Kunst des Arztes keineswegs etwas Fremdes war und im Gegenzug auch manche Ärzte ihre Kunst philosophisch unterfütterten.

Wir behaupten daher, dass das antike Denken über die Entfaltung von Innovation und Wissenschaft eigentlich von der Heilung her zu verstehen ist, d. h. von der Therapie des Gewebes des Lebens, mit dem Ziel, die Harmonie zwischen Mensch und Kosmos wiederherzustellen.

An dieser Stelle können wir nur einige Grundzüge des Verhältnisses der Kunst des Arztes und der des Philosophen skizzieren, um an das antike Motiv des Therapeutischen zu erinnern. Für dieses Anliegen fokussieren wir unseren Blick auf den denkenden Arzt Hippokrates und den ärztlich denkenden Philosophen Sokrates aus Platons Dialogen.

\section{Der denkende Arzt und das ärztliche Denken - einführende Überlegungen}

Betrachten wir zunächst die grundlegenden Motive, die der antike Arzt und der Philosoph des alten Griechenlands teilen. Das Moment des Philosophischen ist die kritische Haltung, das entsprechende Handeln ist das genaue Unterscheiden, mit dem Ziel, das aufzudecken, was ist, was verdeckt wurde und in Vergessenheit geriet: Das philosophische Moment entlarvt das Falsche und Täuschende. Dagegen war zuvor, im kosmischen Wahrheitsverhältnis, der Weg zum Einklang von Wahrem und Täuschendem noch möglich (z. B. bei Heraklit).

Auch die antiken Ärzte wollen die Krankheit aus dem Versteckten locken, sie entlarven und aus der Verbergung holen. Sie wollen die Disharmonie im Körper aufzeigen und alles Falsche und Täuschende ausschließen. Das Ziel ist auch hier die Wahrheit. Diese ist für den Arzt die Gesundheit (hygieia) - und zwar qua prognostischer Sorge und Heilung (therapeia). Für den Arzt ist der Einklang (harmonia) dann erreicht, wenn die Gesundheit wiederhergestellt ist. Der Mensch ist wieder mit der Wahrheit eins, wenn alles Falsche aus dem Körper oder der Seele genommen ist. Auch der Arzt muss hinsichtlich der Wahrheit der Krankheit genau unterscheiden, um die wahre Gesundung einzuleiten und die Harmonie von Körper und Geist wiederherzustellen.

Der Arzt sucht die Verwobenheit mit der Weltordnung und dem Weltganzen wiederherzustellen, indem er den Körper heilt und somit in Einklang mit dem Kosmos bringt. Nur wer geheilt ist, ist im Körperlichen im Einklang, und nur so kann er mit klarem und nicht abgelenktem Verstand die Wahrheit sehen und sich das Vergessene wieder aneignen. Es gibt also in den Ansätzen der frühen Philo- 
sophen und Ärzte eine Synthese von Körper und Geist, von Therapie und Wahrheitssuche, die dem Menschen helfen soll, wieder mit der Weltordnung eins zu werden. Denn sowohl der antike Wahrheits- als auch der Therapiebegriff suggerieren, dass einmal ein harmonischer Zustand gegeben war und dieser lediglich verloren, vergessen oder verborgen wurde bzw. der Erkrankung anheimgefallen ist.

Der antike Arzt prognostiziert, nimmt die Anamnese auf, diagnostiziert, und schließlich folgt die Therapie, bis die Genesung erreicht ist. ${ }^{2}$ Dabei gilt es, wie in der Philosophie auch, eine Krise zu überwinden. Der Philosoph hingegen entwickelt eine Hypothese (Vorannahme, Unterstellung) und entfaltet dann eine verbindliche These (Stellungnahme). Sein Verfahren ist die Logik. Ist die Krise qua kritischer Betrachtung überwunden, gibt es analog zur Therapie des Arztes eine klärende Konklusion. Der antike Arzt und der antike Philosoph sind somit die Helfer der Menschen in Notzeiten. Ihre jeweilige Kunst ist im Wechselbezug von Wahrheitsaneignung und Therapie miteinander verwoben. ${ }^{3}$

\section{Zur Genese des Grundverhältnisses zwischen philosophischem Denken und der Kunst des Arztes}

Schauen wir uns das Verhältnis zwischen dem denkenden Arzt und dem ärztlichen Denken des Philosophen etwas näher an. Dazu können wir uns exemplarisch zwei antike Protagonisten vornehmen: Hippokrates von Kos (ca. 460 bis 370 v.Chr.) und Platons Sokrates in Athen (ca. 469 bis 399 v. Chr.). Hippokrates und Sokrates waren in biographischer Hinsicht sehr verschieden. Ersterer war Arzt, Letzterer war Steinmetz und Philosoph. Der eine war Verfasser von prosaischen

2 Mit Prognose ist hier nicht die medizinische Prognose der zukünftigen Krankheitsentwicklung gemeint, sondern die allgemeine Gesundheitsprognose des Patienten durch Einbettung (Verwobenheit) des Patienten in (mit) seine(r) Umwelt.

3 Auch wenn sowohl Sokrates als auch Hippokrates (bzw. die hippokratische Schule) stark vom Konzept des Wechselspiels der Gegensätze in der Tradition Heraklits beeinflusst sind, so ist doch bemerkenswert, dass vor allem die hippokratische Ärztelehre die Wechselbeziehung jenseits der Einheit in einer Vielheit verortete, die dem All bzw. dem Korbgeflecht und dem Tuch gleich seien (Hippokrates 1995 [1933], Die Diät, 4-6, III/29-32; 14, III/39; 19, III/41). Diese Hinweise legen aus heutiger Perspektive eine Interpretation nahe, die das rhizomatische Moment (Deleuze \& Guattari 1977 [1976]) in diesem geschichtlichen Ort inmitten der vordergründing vorsokratischen Prägung sichtbar werden lässt, so wie Gilles Deleuze und Félix Guattari es in ihrer Einleitung zu Mille Plateaux als hermeneutische Deutung nahelegen. 
Abhandlungen, der andere schrieb überhaupt nichts auf und seine Lehren sind uns nur in der Kunstform der philosophischen Dialoge durch die Sichtweise seines Schülers Platon überliefert. Der eine lebte auf einer eher abseits gelegenen Insel, der andere im griechischen Zentrum Athen. Beide hatten jedoch einen ähnlichen Stil des Denkens, den wir die Verwobenheit des ärztlichen mit dem philosophischen Denken nennen wollen.

\subsection{Hippokrates' Prognosen}

Hippokrates ist das Paradebeispiel eines denkenden Arztes. Er ist für seinen ärztlichen Eid bekannt, der über die Geschichte in abgewandelter Form bis heute seine sinngemäße Gültigkeit bewahrt hat und auch in der Gegenwart der ethische Leitfaden für Ärzte geblieben ist (Flashar 2016, S. 36 ff.). Dabei ist der dogmatisch formulierte Eid des Hippokrates eine seiner geringsten Leistungen, vor allem im Vergleich zu seinen großen medizinischen Arbeiten, die er als einer der Ersten seiner Zunft in Form von Prosatexten angefertigt hat. Mit einer Systematik und Argumentationskraft, die nach ihm erst wieder der Philosoph Aristoteles erreicht hat, sehen wir einen denkenden Arzt am Werk. Dazu betrachten wir zwei Texte aus dem hippokratischen Schriftkorpus, die wahrscheinlich echte Vorlesungen von ihm sind. Diese sind die Abhandlung Luft, Wasser und Ortslage (Über die Umwelt) sowie das kleine Büchlein Prognostikon (Flashar 2016, S. 33-34).

Hippokrates eilt in beiden Texten der Krankheit durch Prophylaxe und Prävention voraus, indem er durch zahlreiche Indizien versucht, ein Vorauswissen (prognôsis) darüber zu gewinnen, was möglichen zukünftigen Patienten passieren kann. Dies vollzieht er in beiden Schriften in einer vorbildlichen, philosophischen Weise, indem er die konkreten Verweisungszusammenhänge der Menschen berücksichtigt (siehe Abb. 1 A). Der Mensch wird weder im Prognostikon noch in der Schrift Luft, Wasser und Ortslage isoliert betrachtet. Es gibt hier weder eine logisch-sprachliche Abstraktion in Bezug auf Benennungen und Tätigkeiten, wie in der späteren Antike, noch eine (mittelalterlich-neuzeitliche) Vermittlung zwischen Subjekt und Objekt, vielmehr wird das Gewebe der Lebensverhältnisse selbst im Hinblick auf den möglichen zukünftigen Patienten oder eine Patientengruppe in Augenschein genommen. In Luft, Wasser und Ortslage heißt es gleich zu Beginn:

\footnotetext{
Wer die Heilkunst in rechter Weise zu erlangen suchen will, hat folgendes zu tun. Zuerst muß er erwägen, welche Wirkung jede einzelne Jahreszeit auszuüben vermag [...]. Alsdann muß er auf die Winde, auf die warmen wie auf die kalten achten, und zwar insbesonders auf die, von welchen alle Menschen beeinflußt werden, nächstdem aber auch auf die, welche jedem Land
} 


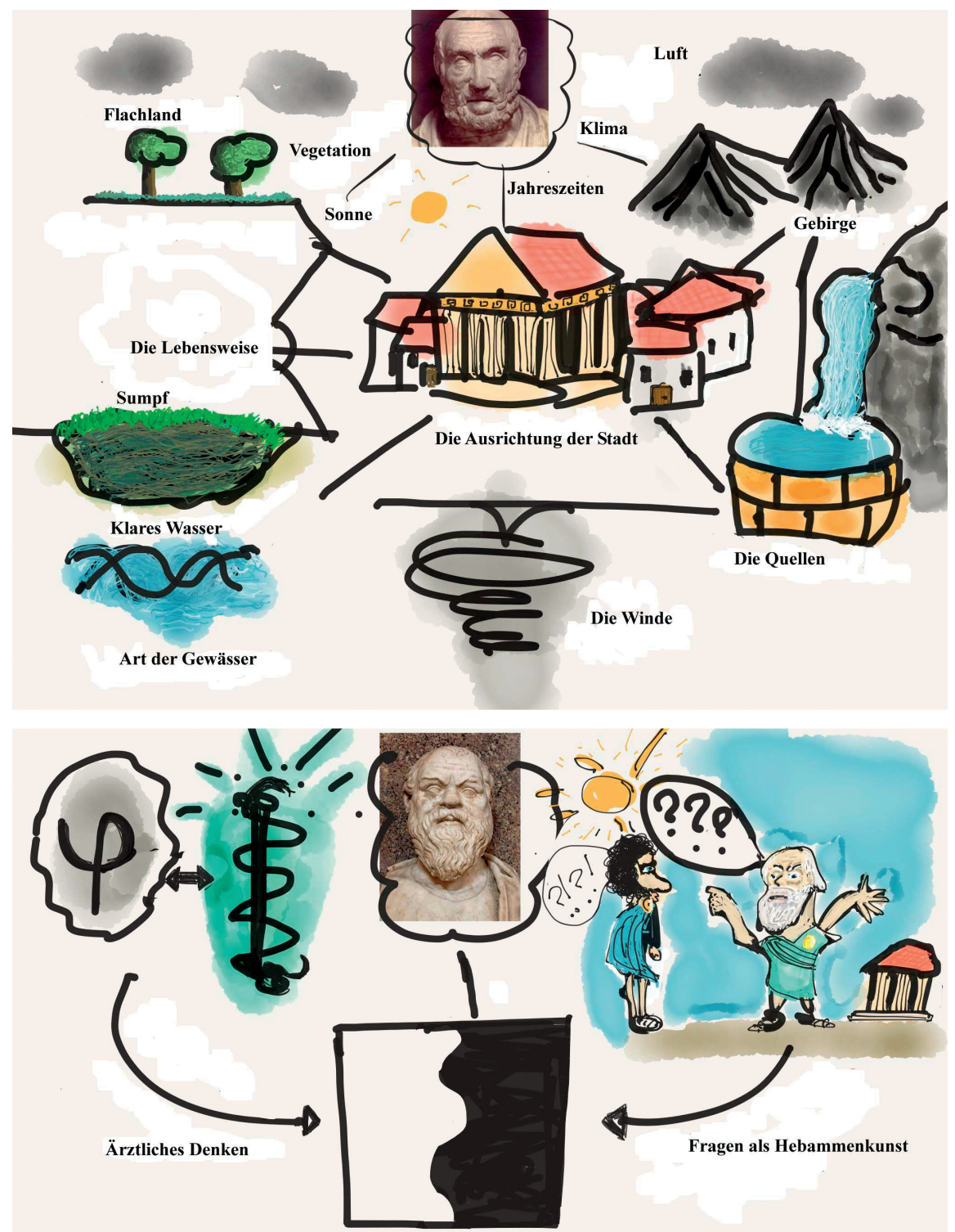

Wege zur Wahrheit und zum Einklang

Abb. 1A (oben): Hippokrates' Verweisungszusammenhang der Gesundheitsprognose Abb. 1B (unten): Sokrates' ärztliches Denken 
eigentümlich sind. Er muß sodann auch die wirksamen Eigenschaften der Gewässer in Betracht ziehen; denn so wie sie sich im Geschmack und Gewicht unterscheiden, so ist auch die Wirkung jedes einzelnen Wassers ganz verschieden (Hippokrates 1995 [1933], Kap. 1, VI/23).

Bereits hier zeichnet sich ab, dass der Mensch immer schon als ein in die Umwelt eingebettetes Wesen verstanden wird. Dies wird noch deutlicher, wenn Hippokrates folgert:

Man muß demnach, wenn man in eine Stadt kommt, die man nicht kennt, genau darauf achten, welche örtliche Lage sie einerseits zu den Winden, andererseits zu den Sonnenaufgängen hat. Eine nach Norden zu gelegene Stadt übt nämlich auf den Menschen nicht denselben Einfluß aus wie eine nach Süden zu gelegene und eine nach Sonnenaufgang zu gelegene nicht denselben Einfluß wie eine nach Sonnenuntergang zu gelegene Stadt (Hippokrates 1995 [1933], Kap. 1, VI/23).

$\mathrm{Zu}$ achten hat man „ferner auf die Gewässer, wie (die Bewohner mit ihnen) daran sind, ob sie sumpfiges, weiches oder hartes, sei es von hochgelegenen und gebirgigen Gegenden kommendes oder sei es salziges und nicht zu erweichendes Wasser haben“ (Hippokrates 1995 [1933], Kap. 1, VI/23). Der Laie, aber auch der reguläre Mediziner der Gegenwart würden diese Aspekte für den Patienten vielleicht vernachlässigen. Für Hippokrates jedoch sind diese Verweisungszusammenhänge für die menschliche Gesundheit unabkömmlich.

Mit Akribie betrachtet Hippokrates diese Zusammenhänge in Bezug auf Klima und Vegetation eines Landes oder einer Region (Hippokrates 1995 [1933], S. VI/ 23-VI/24). Damit aber nicht genug: „Und dann hat man die Lebensweise, welche die Bewohner bevorzugen, ins Auge zu fassen, ob sie gern trinken, nur einmal essen und sich nicht plagen oder ob sie körperliche Bewegung und Arbeit lieben und dabei gern essen, aber nicht trinken“ (Hippokrates 1995 [1933], S. VI/24). Der heutige Mediziner achtet ebenfalls auf die Sportlichkeit der Menschen. Und wenn er das konkrete Lebensumfeld des Patienten berücksichtigt, wird auch er auf die Wasserqualität einer Region achten, wenn die Beschwerden des jeweiligen Patienten auf ein diesbezügliches Problem hinweisen. Hippokrates' Herangehensweise ist auch heute noch vorbildlich, denn er beachtet deutlich mehr Aspekte, als es gegenwärtig vielleicht üblich wäre. Er sieht das gesamte Gewebe des Patienten, aber nicht erst bei der Anamnese oder der Symptom-Behandlung - nein, er prognostiziert für den Einzelnen, für eine Gruppe oder eine Stadt den Gesundheitszustand im umgebenden Kontext (Umwelt, Lebensverhältnisse; siehe Abb. 1 A). Diese Verweisungszusammenhänge zu beachten und aufzuzeigen, bemühen sich die Gesundheitswissenschaften (Public Health), sie werden aber auf der Ebene des Individuums (Patienten) in der praktizierten Allgemeinmedizin 
häufig nicht genügend berücksichtigt. Hippokrates hingegen lässt hinsichtlich deren Wichtigkeit keinen Zweifel offen:

\begin{abstract}
Auf jede einzelne der genannten Eigenschaften hat man also zu achten. Demjenigen nämlich, welcher dies alles, oder wenigstens fast alles genau kennt, wird es, wenn er in eine ihm unbekannte Stadt kommt, weder verborgen bleiben können, welche Krankheiten dem Land eigentümlich sind, noch wie die innere Konstitution (der Menschen) ist. Folglich wird er bei der Behandlung der Krankheiten weder in Verlegenheit kommen noch in die Irre gehen, was natürlicherweise geschehen muß, wenn man diese (Verhältnisse) vorher weder kennen gelernt noch im Einzelnen bedacht hat (Hippokrates 1995 [1933], S. VI/24).
\end{abstract}

Dabei legt es Hippokrates neben der räumlichen Dimension auch im Hinblick auf die Zeit auf maximale Präzision an. „Im Lauf der Zeit wird man ferner über das Jahr aussagen können, sowohl was für allgemeine (epidemische) Krankheiten die Stadt entweder im Sommer oder im Winter heimsuchen werden, als auch welche Krankheiten einem jeden Menschen persönlich bei (einer) Änderung seiner Lebensweise drohen“ (Hippokrates 1995 [1933], S. VI/24). Anschließend warnt Hippokrates, am Beispiel der Bedeutsamkeit und der Einflüsse des Wetters und der Astronomie auf den Menschen, ganz entschieden vor jeder leeren Abstraktion von Krankheiten jenseits der konkreten Lebensverhältnisse des Patienten.

Wer nämlich Bescheid weiß über die Veränderungen der Jahreszeiten und den Auf- und Untergang der Gestirne [...], der wird voraussehen können, wie sich das Jahr gestalten wird. Daher wird einer, der hiernach forscht und die (entscheidenden) Zeitpunkte im voraus erkennt, über jede Einzelheit genau Kenntnis haben, er wird sowohl die beste Gesundheit erzielen, wie auch vor allem in der (Heil-)kunst den richtigen Weg einschlagen. Sollte aber einer der Ansicht sein, dass diese Fragen nur in das Gebiet der Meteorologie gehören, so dürfte er, sobald er seine Meinung ändert, merken, dass die Astronomie der ärztlichen Kunst keine geringen, sondern sehr wesentliche Dienste leistet. Denn zugleich mit dem Klima (hôrai) ändern sich bei den Menschen die Leibeshöhlen (das Innere) (Hippokrates 1995 [1933], S. VI/24-VI/25).

Bereits hier wird deutlich, dass Hippokrates nicht nur leere Behauptungen aufstellt. Auch in den folgenden Kapiteln seiner Schrift entfaltet er in strukturierter Form Begründungen und Erläuterungen seiner Thesen. Diese vorbildlichen und umfangreichen Einsichten eines antiken Arztes konnten in jener Zeit nur von einem Menschen gewonnen werden, der nicht nur praktiziert, d.h. die Kunst des Arztes anwendet, sondern sich in sie auch therapeutisch hineindenkt. Hippokrates ist der Erste, der schriftlich auf den Zusammenhang der Kunst des Arztes in seiner Praxis mit dem Verweisungszusammenhang von Umwelt und Mensch hingewiesen hat. Ärztlich-therapeutisch dachte auch der philosophierende Zeitgenosse des Hippokrates von Kos: Platons Sokrates. 


\subsection{Sokrates’ Mäeutik in der Elenktik}

Platon lässt Sokrates in dreifacher Weise über medizinische Praxis denkend sprechen: Erstens ist die Gesprächstechnik (Elenktik) des Sokrates selbst so etwas wie ein ärztlicher Vorgang, wenn er im Dialog Theätet der Wahrheit zur Geburt verhelfen will, und zweitens vergleicht er im Dialog Phaidros die Arbeit des Philosophen mit der des Arztes (vgl. Abb. 1B). Drittens liegt mit der zweiten Hälfte des Dialogs Timaios eine philosophisch-ärztliche Abhandlung vor. Darauf wollen wir ein Schlaglicht werfen. Im Theätet macht Sokrates auf das medizinische Wissen der Hebammen aufmerksam: „Und können die Hebammen nicht auch durch Verabreichungen von Mittelchen [...] die Schmerzen erregen und sie nach Belieben auch mildern und bei Schwergebärenden die Geburt ermöglichen [...]. Theät. So ist's“ (Platon 1998 [1919], Bd. 4, Theait., S. 41, St. 149d 1-5). Sokrates selbst nennt seine Fragetechnik eine Hebammenkunst (Mäeutik) - allerdings versteht er sie nicht als ärztliche Technik für die Geburt des Körpers, sondern für die des Geistes (vgl. Abb. 1B). So sagt er über die Hebammen:

\footnotetext{
Mit meiner Entbindungskunst steht es nun im übrigen wie bei jenen [...]. Der wichtigste Teil aber meiner Kunst ist die Fähigkeit, auf jede Weise zu prüfen, ob der Geist des Jünglings eine Schein- und Lügengeburt zutage bringt, oder etwas Echtes und Wahres. Denn in folgendem Punkt gleiche ich ganz den Hebammen: [...] mit dem Vorwurf, den schon viele mir gemacht haben, dass ich nämlich zwar die anderen frage, selbst aber keinerlei Antwort gebe, weil ich über keine Weisheit gebiete, hat es seine volle Richtigkeit. Der Grund davon ist folgender: zu entbinden zwingt mich der Gott, selbst aber zu gebären hat er mir versagt. [...] Die Entbindung aber ist des Gottes und mein Werk (Platon 1998 [1919], Bd. 4, Theait., S. 42-43, St. $150 b-c)$.
}

Dies erinnert uns an die Worte des Sokrates vor den Anklägern - „Ich weiß, dass ich nichts weiß“ - aus der Apologie (Platon 1998 [1919], Bd. 1, Apol., S. 29 ff, St. $21 \mathrm{ff}$.). Wie der Arzt sich nicht selbst heilen kann, so kann auch die Hebamme sich nicht selbst entbinden helfen. Auch hier gibt es eine Parallele zum Philosophen, der sich selbst nicht weise machen kann: Nur im Dialog gelingt es, gemeinsam zur Weisheit zu gelangen, wobei der Philosoph mittels eines ärztlichen Denkens agiert.

Dass dies Platons Überzeugung ist, lässt sich am Dialog Phaidros zeigen. Dort weist Sokrates den Phaidros zurecht, der glaubt, die Wahrheit zähle überhaupt nicht, sondern einzig die Kunst des Überredens und der Rhetorik sei von Bedeutung: „Heran denn, ihr edlen Weisen, und überredet den Phaidros, den Vater schöner Kinder, daß, wenn er nicht tüchtig Philosophie treibt, er auch niemals imstande sein wird, tüchtig über irgendeinen Gegenstand zu reden“ (Platon 1998 [1919], Bd. 2, Phdr., S. 80, St. 261a). So vergleicht Sokrates denjenigen, der redet, 
ohne zu philosophieren, mit einem Quacksalber - und dies lässt er Phaidros selbst entwickeln: „Weil er aus irgendeinem Buche darüber hat vorlesen hören oder Arzneien in die Hand bekommen hat, bildet er sich ein, ein Arzt zu sein, während er doch nichts versteht von der Kunst“ (Platon 1998 [1919], Bd. 2, Phdr., S. 92, St. 268c1-4). Sokrates schärft Phaidros aber ein:

Die Weise der rednerischen Kunst [insofern sie philosophisch ist und auf die Seele des Menschen abzielt] ist doch wohl dieselbe wie die der ärztlichen. [...] In beiden musst du die Natur unterscheiden, dort des Körpers, hier der Seele, wenn du nicht allein nach Handwerksgebrauch und Erfahrung, sondern nach Kunst willst jenen Gesundheit und Kraft einflößen durch Darreichung von Arzneien und Nahrung, dieser eine beliebige Überzeugung beibringen und Tüchtigkeit durch Worte und Übung geordneten Verhaltens (Platon 1998 [1919], Bd. 2, Phdr., S. 95, St. 270b 4-9, Hinzufügung von M. M.).

Direkt und explizit beziehen sich Phaidros und Sokrates dabei auf Hippokrates und zwar so, dass sie Hippokrates dafür loben, dass er (wie oben bereits erläutert) den Verweisungszusammenhang von Mensch und Welt sowie deren gemeinsame Verwebung mit der Gesundheit beachtet:

Sokrates. Glaubst du nun, es sei möglich, von der Natur der Seele eine nennenswerte Kenntnis zu erwerben ohne Zusammenhang mit der Natur des Ganzen der Welt?

Phaidros. Wenn man auf Hippokrates aus dem Geschlecht der Asklepiaden sich einigermaßen verlassen darf, wäre das nicht einmal bezüglich des Körpers möglich ohne diese Betrachtungsweise.

Sokrates. Und er hat Recht, mein Freund. Doch müssen wir außer dem Hippokrates auch die Vernunft fragen und sehen, ob sie damit übereinstimmt (Platon 1998 [1919], Bd. 2, Phdr., S. 95-96, St. 270c 1-8).

Mithilfe der Vernunft und der Lehre des Hippokrates von der ärztlichen Kunst versucht Sokrates nun zusammen mit Phaidros zu einer genauen Differenzierung bezüglich der Natur - sei es die der Seele oder die des Körpers - zu gelangen. „Wenigstens gliche jedes Verfahren, das davon absieht, wohl fast der Wanderung eines Blinden, während hingegen der mit Kunst irgendeine Sache Betreibende [mit] keinem Blinden oder Tauben verglichen werden darf“ (Platon 1998 [1919], Bd. 2, Phdr., S. 95-96, St. 270c 1-8).

Damit ist deutlich geworden: Das Denken des Arztes und das ärztliche Denken des Philosophen durchdringen einander. Sowohl Sokrates als auch Hippokrates geht es um die Therapie des Menschen - der Philosoph achtet auf die Seele, der Arzt auf den Körper. Dies sehen wir auch in der zweiten Hälfte des Timaios, bevor Timaios das Verhältnis der ärztlichen und philosophischen Herangehensweise verquickt und über die Seele (den Geist) und den Körper sagt: „Es gibt nur eine Rettung für beide: die Seele darf nicht ohne den Körper und der Körper nicht 
ohne die Seele in Bewegung gesetzt werden, auf daß beide, ihre Rechte verteidigend, ins Gleichgewicht und zur Gesundheit gelangen“ (Platon 1998 [1919], Bd. 6, Tim., S. 136, St. 88d 1-3). Entsprechend durchdringen für Platons Timaios die Kunst des Arztes und die Philosophie einander, um dem Ideal des Ebenmaßes (der Symmetrie) von Wahrheit, Schönheit und dem Guten zu entsprechen (Platon 1998 [1919], Bd. 6, Tim., S. 134-135, St. 87).

\section{Mit den antiken griechischen Denkern auf dem Weg zu einem europäischen Gesundheitsbegriff der Zukunft}

Die Gesundheitswissenschaften (Public Health) sind eine Multidisziplin aus Epidemiologie, Naturwissenschaften (Biomedizin, Genetik, Umweltwissenschaften), Sozialwissenschaften (Soziologie, Psychologie, Demographie) und Wirtschaftswissenschaften, die sich interdisziplinär mit Gesundheitsfragen befassen und transdisziplinär mit Praxispartnern, Institutionen des Gesundheitssystems und politischen Akteuren zum Wohle der Gesundheit der Bevölkerung zusammenarbeiten. Dabei wird im Unterschied zur Medizin, wo der individuelle Patient mit seiner Krankheit im Vordergrund steht, ein populationsbezogener Ansatz verfolgt, der sich auf die gesamte Bevölkerung eines Landes, der Welt oder auf bestimmte Bevölkerungsgruppen bezieht. Zusätzlich zur Beobachtung (Gesundheitsberichterstattung, Monitoring, Surveillance) und der Charakterisierung von Krankheitslasten werden Gesundheitsdeterminanten identifiziert und laufend beobachtet, die in bestimmter Weise mit Erkrankungen bzw. mit Gesundheit assoziiert sind und zu deren Verminderung bzw. Vermehrung beitragen. Daraus leiten sich Präventionsmaßnahmen ab. Des Weiteren ist die Methodik der Gesundheitswissenschaften systembezogen, weil die Analyse der Einrichtungen des Gesundheitssystems zur fortwährenden Anpassung dieses Systems an geänderte Krankheitslasten beitragen soll, um die Krankheitslasten in der Bevölkerung zu senken, wobei wirtschaftliche Gesichtspunkte berücksichtigt werden.

Charakteristisch für die modernen Gesundheitswissenschaften sind Konzepte wie der Settingansatz, der Lebensstil, Lebenswelten und andere Verweisungszusammenhänge betrachtet, die für die Gesundheit der Menschen entscheidend sind. Bespiele solcher Settings sind Organisationen wie Betriebe, Erziehungseinrichtungen, Einrichtungen des Gesundheitswesens, aber auch größere Einheiten wie Städte (Fehr \& Hornberg 2018), Megastädte (Krämer et al. 2011) und thematische Lebenswelten wie die von Migranten und Flüchtlingen (Krämer \& Fischer 2019), Klimagesundheit im Kontext von Klimawandel sowie 
Infektionen (Krämer et al. 2010) und Pandemien (siehe die gegenwärtige COVID19-Pandemie). Diese Settings bzw. Kontexte ermöglichen die Einbeziehung weiterer wissenschaftlicher Kooperationen z.B. mit den Geowissenschaften, der Stadtplanung und Architektur, der Anthropologie und Ethnologie oder mit der Klimatologie und Mathematik (Algorithmen und Modelle für Monitoring und Vorhersagen von Krankheiten). Der Settingansatz ist auch ein Grundprinzip für die Sustainable Development Goals (SDG) der UN/WHO (Ganten et al. 2018; Griggs et al. 2013; Wikipedia 2020). Wie sollen allerdings diese Disziplinen halbwegs harmonisch und effizient zum Wohle der Gesundheit zusammenarbeiten? Welcher theoretische Rahmen muss geschaffen werden, um für fruchtbringende wissenschaftliche Kooperationen zu sorgen, deren Ergebnisse dann erfolgreich in die Praxis umgesetzt werden können? Welche innovativen theoretischen Prinzipien könnten leitend sein?

Der Rückgriff auf die Philosophie - insbesondere auf das philosophische Denken im antiken Griechenland - könnte den vorhandenen Theoriebedarf, welcher sich in den Health Sciences (Medizin und Public Health) findet, decken und dabei helfen, einen ganzheitlichen Gesundheitsbegriff weiter zu entwickeln und stärker zu fundieren. Dieser Gesundheitsbegriff wäre damit im eigentlichen Sinne ein philosophischer und könnte es ermöglichen, Inhalte und Verfahren von Einzeldisziplinen der Health Sciences und von ihren wissenschaftlichen Partnern $\mathrm{zu}$ integrieren, um so Synergien zu generieren.

Ein solchermaßen philosophisch fundierter Theorieansatz in den Health Sciences wäre bestens dazu geeignet, kurzfristig orientierte Interessenskonflikte (Natur- versus Sozialwissenschaften, Genetik versus Umwelt, Physis versus Psyche, Gesundheit versus ökonomischer Gewinn, prädiktive Medizin versus gesundheitliche Ungleichheit, molekulare Medizin versus Sozialmedizin) in ihrer kräftezehrenden Dichotomie aufzuheben, um die Einzeldisziplinen so synergistisch zusammenzuführen. Auch wenn es illusorisch ist und überdies auch nicht wünschenswert wäre, die bekannten Gegensätze gänzlich aufzulösen, könnte der Rückgriff auf die antike griechische Philosophie hinsichtlich der Verwebung von ärztlicher Kunst und Philosophie inspirieren und zu einem Element und möglicherweise Leitmotiv für einen auch ethisch fundierten Gesundheitsbegriff der Zukunft werden (Stoecker et al. 2011). Dem Öffentlichen Gesundheitsdienst (ÖGD) käme dabei die Aufgabe zu, die integrierende Rolle für die Praxis zu übernehmen. Es ist mehr als bemerkenswert, wie weitsichtig insbesondere Hippokrates - ohne Ablenkung durch kapitalistische, sozialistische oder andere ideologische Konzepte - in seinen Schriften zentrale Konzepte der heutigen Gesundheitswissenschaften wie etwa Lebensstil, Lebensverhältnisse, Setting und Umwelt (z. B. Klima) - also einen allgemeinen Weltbezug - mitgedacht hat. 
Vielleicht könnte die Rückbesinnung auf die von den antiken griechischen Philosophen begründete europäische Denktradition (Eckart 2013) dazu beitragen, dass sich das europäische Denken vor dem Hintergrund einer zerfallenden politischen Weltordnung gegen den mit diesem Zerfall einhergehenden Theorie- und damit Sinnverlust auf geistigem und intellektuellem Terrain neu $\mathrm{zu}$ formieren lernt und dass diese Besinnung bereits in naher Zukunft nutzbar gemacht werden kann. In der Zwischenzeit wünschen wir uns, dass die hier skizzierten Überlegungen für eine weiterentwickelte Theorie der Health Sciences Verwendung finden.

\section{Literatur}

Deleuze, Gilles/Guattari, Félix (1977 [1976]): Rhizom (Dagmer Berger/Clemens-Carl Haerle/Helma Konyen/Alexander Krämer/Michael Nowak/Kade Schacht, Übers.). Berlin: Merve.

Eckart, Wolfgang U. (2013): Geschichte, Theorie und Ethik der Medizin. Heidelberg: Springer. Fehr, Rainer/Hornberg, Claudia (Hrsg.) (2018): Stadt der Zukunft - Gesund und nachhaltig: Brückenbau zwischen Disziplinen und Sektoren. München: Oekom.

Flashar, Hellmut (2016): Hippokrates. Meister der Heilkunst. Leben und Werk. München: C. H. Beck.

Ganten, Detlev/Silva, João Gabriel/Regateiro, Fernando et al. (2018): „Science Has to Take Responsibility. 10 Years World Health Summit - The Road to Better Health for All“. In: Front Public Health 6, S. 314.

Griggs, David/Stafford-Smith, Mark/Gaffney, Owen et al. (2013): „Sustainable Development Goals for People and Planet“. In: Nature 495(7441), S. 305-307.

Hesiod (1996): Werke und Tage (Otto Schönberger, Hrsg.). Stuttgart: Reclam.

Hippokrates (1995 [1933]): „Luft, Wasser und Ortslage“. In: Richard Kapferer (Hrsg.): Sämtliche Werke, Bd. 6. Anger: Eick, $\mathrm{VI} / 5-58$.

Krämer, Alexander/Fischer, Florian (Hrsg.) (2019): Refugee Migration and Health: Challenges for Germany and Europe. Cham: Springer International.

Krämer, Alexander/Khan, Mobarak Hossain/Kraas, Frauke (Hrsg.) (2011): Health in Megacities and Urban Areas. Heidelberg: Springer.

Krämer, Alexander/Kretzschmar, Mirjam/Krickeberg, Klaus (Hrsg.) (2010): Modern Infectious Disease Epidemiology: Concepts, Methods, Mathematical Models, and Public Health. New York, NY: Springer International.

Platon (1998 [1919]): Sämtliche Dialoge (Otto Apelt, Hrsg.). Hamburg: Meiner.

Stoecker, Ralf/Neuhäuser, Christian/Raters, Marie-Luise (Hrsg.) (2011): Handbuch Angewandte Ethik. Stuttgart: Metzler.

Wikipedia (2020): „Ziele für nachhaltige Entwicklung“. https://de.wikipedia.org/wiki/Ziele_f\% C3\%BCr_nachhaltige_Entwicklung, besucht am 21.04.2020. 